\title{
Simplifying LR-HSQC Spectra using a Triple- Quantum Filter: The LR-HTQC Experiment
}

\author{
Peter Bigler*, Julien Furrer* \\ Departement für Chemie und Biochemie, Universität Bern, \\ Freiestrasse 3, CH-3012 Bern, Switzerland
}

*biglermeier@bluewin.ch (PB)

*julien.furrer@dcb.unibe.ch (JF) 
Abstract Long-Range Heteronuclear Single Quantum Correlation (LR-HSQC) experiments may be applied for detecting long-range correlations, but suffer from two disadvantages, common to all heteronuclear long-range correlation experiments: (i) The information density in LR-HSQC spectra may be too high to be used directly without "filtering out" shorter range correlations, and (ii) often, substantial differences in intensity among cross peaks exist, potentially hampering the visualization of weak, often crucial cross peaks. In this contribution, we propose a modified LR-HSQC experiment, the LR-HTQC experiment (Long-Range Heteronuclear Triple Quantum Correlation) that partially solves the problems aforementioned. We show theoretically and experimentally that the LR-HTQC experiment removes the intense cross peaks of $\mathrm{CH}$ spin pairs, substantially reduces the medium intensity of cross peaks originating from $\mathrm{CHH}^{\prime}$ spin systems, while the typically weak intensity of cross peaks of $\mathrm{CHH}$ 'H" and $C(H)_{n}, n>3$ spin systems is less affected. Consequently, the LR-HTQC experiment affords simplified long-range heteronuclear shift correlation spectra and scales down large intensity differences among different types of cross peaks, although a certain general reduction of signal intensities has to be accepted.

Keywords: NMR, ${ }^{1} \mathrm{H},{ }^{13} \mathrm{C}$, LR-HSQC, LR-HTQC, spectral simplification, cross peak intensity, product operator. 


\section{Introduction}

Heteronuclear long-range correlation experiments correlate protons and heteronuclei exploiting ${ }^{n} J_{H X}$ long-range couplings. The experiments are essential to connect structural fragments across non-protonated carbons or heteroatoms. ${ }^{[1-9]}$ Prior to the introduction of proton-detected NMR methods, experiments such as long-range HETCOR and FLOCK were used for this purpose. ${ }^{[10]}$ Currently, there are a plethora of proton-detected methods available for long-range heteronuclear shift correlation, [10-13] with the basic $\mathrm{HMBC}^{[14]}$ as the most commonly used. As an alternative to HMBC experiments long-range versions of HSQC[15-17] may be applied, but have obviously never enjoyed popularity for that purpose. Yet, HSQC-based techniques are expected to be inherently more efficient, since no $J_{\mathrm{HH}}$ modulation occurs during the evolution period $t_{1}$. Hence, a constant-time setting as applied with HMBC to eliminate $J_{H^{\prime}}$ modulation is not necessary. Of note, selective and non-selective versions of the LRHSQMBC pulse sequence, a refocused variant of the LR-HSQC experiment, have been recently proposed and proved to be the best choice for detecting very weak longrange correlations. $\left.{ }^{[4,}, 9,18-23\right]$

However, and despite the absence of $J_{\mathrm{HH}}$-modulation our recent findings demonstrate that in LR-HSQC experiments the intensity of the long-range cross peaks is influenced in an unwanted way by the magnitude and number of passive homonuclear proton-proton couplings $\mathrm{JHH}{ }^{[24]}$ In addition there are two other, long known, disadvantages associated with LR-HSQC spectra: (i) the information density in LR-HSQC spectra due to the large number of detectable ${ }^{n} J_{C H}$ correlations can be too high and therefore confusing. Filters adjusted e.g. for detecting only ${ }^{3} J_{\mathrm{CH}}$ and large ${ }^{2} \mathrm{~J}_{\mathrm{CH}}$ couplings would be advantageous. (ii) Often in LR-HSQC spectra, large intensity differences among cross peaks exist, hampering the visualization of weak, but crucial cross peaks. However, because the intensity of cross peaks depends on the number of protons involved, the number of passive homonuclear couplings $\mathrm{JHH}_{\mathrm{H}}$ and the size of the heteronuclear coupling constant ${ }^{n} J_{C H}$, this problem is rather difficult to be handled.[24]

In this contribution, we propose a variant of the LR-HSQC experiment, the LRHTQC experiment (Long-Range Heteronuclear Triple Quantum Correlation) that partially overcomes these disadvantages of the LR-HSQC experiment. We found theoretically and experimentally that a) the most intense cross peaks of $\mathrm{CH}$ spin pairs 
(i.e. the ${ }^{1} \mathrm{H}$ resonances that appear as singlets in the ${ }^{1} \mathrm{H}$ NMR spectrum, typically methyl resonances) vanish in LR-HTQC spectra, and that b) the intensity of cross peaks of $\mathrm{CHH}$ ' spin systems $\left({ }^{1} \mathrm{H}\right.$ resonances that appear as doublets in the ${ }^{1} \mathrm{H}$ NMR spectrum) is substantially reduced, while the intensity of those cross peaks of interest, i.e. of $\mathrm{C}(\mathrm{H})_{n}, \mathrm{n}>3$ spin pairs is less affected. Therefore, the LR-HTQC experiment eliminates the most intense cross-peaks (of no interest) and scales down the often large intensity differences among different types of cross-peaks. It affords simplified long-range heteronuclear shift correlation spectra and allows the usually weak crosspeaks of interest to be recognized and detected more easily, although a minor yet acceptable general intensity decrease has to be accepted.

\section{Results and Discussion}

\section{LR-HTQC experiment}

The LR-HTQC pulse sequence differs from the LR-HSQC pulse sequence in two points (Figure 1) ${ }^{[15,16]}$ : (i) the purge gradient after the second $90^{\circ} y^{1} \mathrm{H}$-pulse is omitted and (ii) the gradient ratio $\mathrm{G} 1: \mathrm{G} 2$ is set for selecting triple quantum coherences. In the following the discussion is restricted to $\mathrm{CH}_{n}$ spin systems, but the different conclusions are valid for other $\mathrm{XH}_{n}$ spin systems in general. Of note, a ${ }^{15} \mathrm{~N} \mathrm{HTQC-experiment}$ (HmQC) has been proposed by Schmidt and Rüterjans, $\left.{ }^{25,}, 26\right]$ which allows one to detect heteronuclear spin systems of the type $\mathrm{I}_{2} \mathrm{~S}$ exclusively. This ${ }^{15} \mathrm{~N}$ HTQCexperiment is basically a time-reversed DEPT-type sequence ${ }^{[27]}$ that allows the conversion of triple-quantum coherences into detectable single-quantum terms. Of note, a HQQC (Heteronuclear Quadruple-Quantum Correlation) experiment for selecting exclusively the methyl resonances in heteronuclear correlation spectra was also proposed.[28] A related family of experiments has also been reported under the acronym MAXY (MAXimum-quantum correlation spectroscopY). ${ }^{[29]}$ In these experiments, a classical COSY, TOCSY or NOESY building block is appended to the conventional HmQC pulse train,[25] and, for instance, a 2D COSY spectrum showing only proton resonances belonging to $\mathrm{I}_{2} \mathrm{~S}$ systems can be obtained.[29] Finally, this HmQC pulse sequence element - instead of the conventional INEPT block - was used as an initial building block in triple-resonance experiments in various MUSIC (Multiplicity Selective In-phase Coherence transfer) experiments. ${ }^{[30,31]}$ The idea of the 
MUSIC element is to selectively excite $\mathrm{XH}_{2}$ or $\mathrm{XH}_{3}$ spin systems to detect amino-acid selective $2 \mathrm{D}{ }^{1} \mathrm{H}-{ }^{15} \mathrm{~N}$ correlations.

Griesinger at al. later introduced a CT-HTQC experiment (Constant Time Heteronuclear Triple-Quantum Correlation) for detecting $\mathrm{CH}$ and $\mathrm{CH}_{2}$ correlations with its sensitivity enhanced by a factor of up to 3 relative to HSQC spectra in ${ }^{13} \mathrm{C},{ }^{15} \mathrm{~N}$ labeled RNA oligonucleotides. ${ }^{[32]}$

The proposed LR-HTQC uses a similar idea, and allows one to finally detect single quantum coherences of heteronuclear long-range coupled protons, which originates exclusively from intermediate triple quantum coherences.

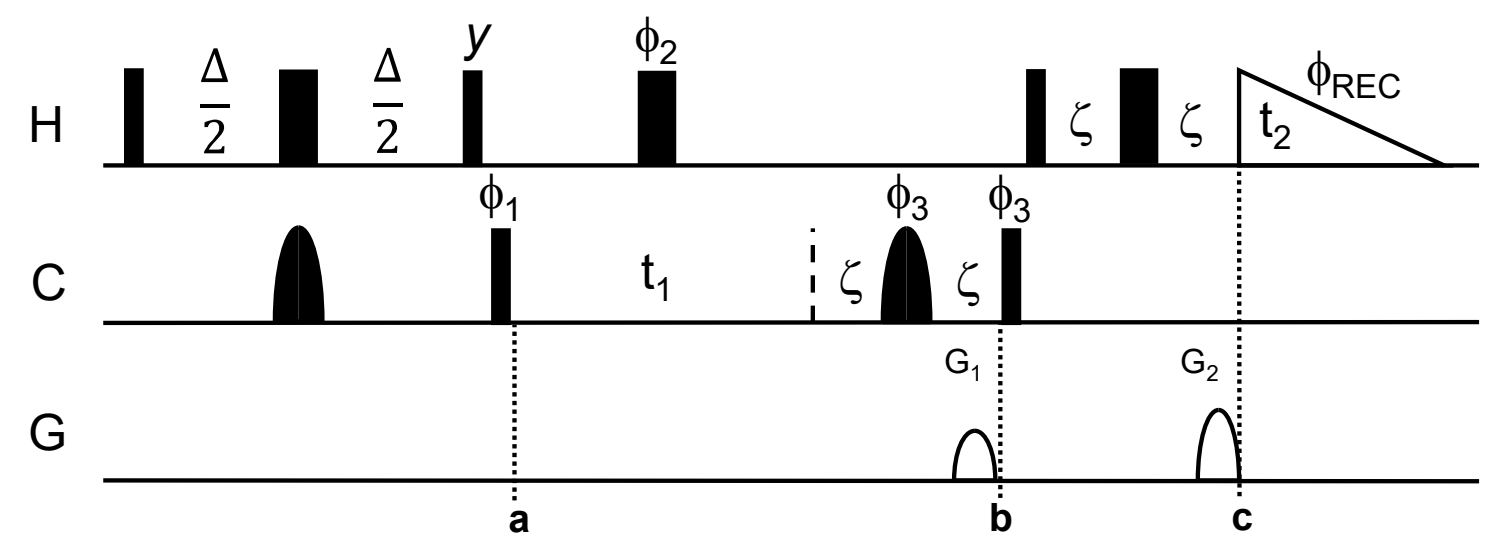

Figure 1. Pulse sequence of the LR-HTQC experiment without a low-pass filter. Thin bars represent $90^{\circ}$ pulses, thick bars $180^{\circ}$ pulses. All ${ }^{13} \mathrm{C} 180^{\circ}$ pulses are adiabatic CHIRP pulses ${ }^{[33]}$ for broadband inversion and refocusing respectively, shown as sine shapes. $\Delta$ is the long-range coupling evolution delay and is set to an average value $0.5 / n J_{C H a v}$. Delay $\zeta$ is set to guarantee proper chemical shift refocusing and is equal to the length of the gradient pulse $G+$ the delay for gradient recovery. The following phase cycling is applied: $\phi_{1}=x,-x ; \phi_{2}=x, x,-x,-x ; \phi_{3}=4 x, 4(-x) ; \phi r e c=x,-x, x,-x,-x, x$, $-x, x$. Phases not shown are applied along the $x$-axis. Gradient ratios of $\mathrm{G}_{1}: \mathrm{G}_{2}=20: 35$ or 20:45 (See Supplementary Material) select triple quantum coherences. The labels a-c indicate the three steps of interest in the pulse sequence.

At stage a, a product operator evaluation for a $\mathrm{CH}$, a $\mathrm{CHH}$ ' and a $\mathrm{CHH}$ 'H" spin system, assuming that all ${ }^{1} \mathrm{H}$ chemical shifts are refocused, yields the following coherences $\left(\mathrm{H}^{r}\right.$ is a proton long-range coupled $\left({ }^{n} \mathrm{~J}_{\mathrm{CH}}\right)$ to carbon $\mathrm{C}$ and $\mathrm{H}^{\prime}$ is a proton coupled to $\mathrm{H}$ through $\mathrm{J}_{\mathrm{HH}}$, with ${ }^{n} J_{\mathrm{CH}}$ and $\mathrm{J}_{\mathrm{HH}} \neq 0$ ). The subscript $r$ will be used subsequently throughout the whole manuscript to indicate the ${ }^{n} J_{C H}$ coupling. Note that there is no difference whether ${ }^{n} J_{C H}$ is zero or not. The reason is that both spins $C$ and 
$H^{\prime}$ are present as $z$-magnetization during $\Delta$, and therefore the $J$-coupling between them remains inactive.

\section{Stage a}

\section{CH spin system}

$H_{Z}^{r} \stackrel{(90)^{\circ} x H}{\longrightarrow} \stackrel{\pi^{n} J_{C H} \Delta 2 H_{Z} C_{Z}}{\longrightarrow} \stackrel{(180)^{\circ} x H}{\longrightarrow} \stackrel{(180)^{\circ} x C}{\longrightarrow} \stackrel{(90)^{\circ} y H}{\longrightarrow} \stackrel{(90)^{\circ} x C}{\longrightarrow} H_{Y}^{r} \cos \left(\pi^{n} J_{C H} \Delta\right)-2 H_{Z}^{r} C_{Y} \sin \left(\pi^{n} J_{C H} \Delta\right)$

As outlined before the proposed LR-HTQC should improve the recognizability of the typically weak signals of additionally $\left(\mathrm{JHH}^{\prime}-\right)$ coupled spins. This is achieved by a so-called triple quantum filter, i.e. by selecting respective coherence pathways - with intermediate triple quantum coherences - with the help of magnetic field gradients. To handle the effect of such gradients - explained below - on the one hand and to simplify the subsequent product operator treatment on the other hand, the individual product operator terms (Cartesian basis) are transformed using the respective raising and lowering operators (spherical basis). They allow at this stage different types of coherences, single quantum (SQ) and triple quantum (TQ), to be recognized, triple quantum coherences to be selected and only their further evolution to be examined.

Thus, for a $\mathrm{CH}$ spin system the $2 \mathrm{H}_{Z}{ }_{Z} \mathrm{C}_{Y}$ term is accordingly transformed into $\mathrm{SQ}$ coherences as follows:

$$
\begin{gathered}
-2 H_{Z}^{r} C_{Y} \sin \left(\pi^{n} J_{C H} \Delta\right) \rightarrow \quad i H_{Z}^{r}\left(C_{+}-C_{-}\right) \sin \left(\pi^{n} J_{C H} \Delta\right) \\
\mathrm{SQ}
\end{gathered}
$$

\section{CHH' spin system}


$\stackrel{(90)^{\circ} x H}{\longrightarrow} \stackrel{\pi^{n} J_{C H} \Delta 2 H_{Z} C_{Z} \pi^{n} J_{H H^{\prime}} \Delta 2 H_{Z} H^{\prime} Z}{\longrightarrow} \stackrel{(180)^{\circ} x H}{\longrightarrow} \stackrel{(180)^{\circ} x H^{\prime}}{\longrightarrow} \stackrel{(180)^{\circ} x C}{\longrightarrow} \stackrel{(90)^{\circ} y H}{\longrightarrow} \stackrel{(90)^{\circ} y H^{\prime}(90)^{\circ} x C}{\longrightarrow} H_{Y}^{r} \cos \left(\pi J_{H H^{\prime}} \Delta\right) \cos \left(\pi^{n} J_{C H}\right.$

$-2 H_{Z}^{r} C_{Y} \cos \left(\pi J_{H H^{\prime}} \Delta\right) \sin \left(\pi^{n} J_{C H} \Delta\right)-2 H_{Z}^{r} H_{X}^{\prime} \sin \left(\pi J_{H H^{\prime}} \Delta\right) \cos \left(\pi^{n} J_{C H} \Delta\right)$

$+4 H_{Y}^{r} H_{X}^{\prime} C_{Y} \sin \left(\pi J_{H H^{\prime}} \Delta\right) \sin \left(\pi^{n} J_{C H} \Delta\right)$

The coherences immediately present after the $90^{\circ} \times{ }^{13} \mathrm{C}$-pulse can be classified as follows: (i) single quantum carbon coherence $2 \mathrm{H}_{Z} \mathrm{C}_{Y}$, (ii) pure proton singlequantum coherences $H_{Y}$ and $2 H_{X}^{r} H_{Z}^{\prime}$ and (iii) a multi-quantum coherence $4 H_{Y}^{r} H_{X}^{\prime} C_{Y}$.

Note that in the LR-HTQC sequence the spoil gradient G3 usually used in the LR-HSQC experiment, ensuring that exclusively coherences generated by polarization transfer will be present during the $t_{1}$-evolution period, must be omitted, to not destroy the desired magnetization term (see below).

The pure proton coherences $H^{r} Y$ and $2 H_{X}^{r} H_{Z}^{\prime}$ can be ignored, since on the one side they will not contribute to carbon coherence for the subsequent ${ }^{13} \mathrm{C}$-shift evolution period $t_{1}$, and on the other side they will be destroyed subsequently by the coherence selection gradients $\mathrm{G} 1$ and $\mathrm{G} 2$.

The single quantum carbon coherence $2 \mathrm{H}_{Z}{ }_{Z} \mathrm{C}_{Y}$ is transformed into $\mathrm{SQ}$ coherences as described above and the $4 H^{r}{ }_{Y} H^{\prime} X C_{Y}$ coherences term are correspondingly transformed into a sum of triple (TQ) and SQ quantum coherences:[34]

$$
\begin{gathered}
-2 H_{Z}^{r} C_{Y} \cos \left(\pi J_{H H^{\prime}} \Delta\right) \sin \left(\pi^{n} J_{C H} \Delta\right) \rightarrow \quad i H_{Z}^{r}\left(C_{+}-C_{-}\right) \cos \left(\pi J_{H H^{\prime}} \Delta\right) \sin \left(\pi^{n} J_{C H} \Delta\right) \\
\mathrm{SQ} \\
4 H_{Y}^{r} H_{X}^{\prime} C_{Y} \sin \left(\pi J_{H H^{\prime}} \Delta\right) \sin \left(\pi^{n} J_{C H} \Delta\right) \rightarrow \\
-0.5\left(H_{+}^{r} H_{+}^{\prime}-H_{+}^{r} H_{-}^{\prime}+H_{-}^{r} H_{+}^{\prime}+H_{-}^{r} H_{-}^{\prime}\right)\left(C_{+}-C_{-}\right) \sin \left(\pi J_{H H^{\prime}} \Delta\right) \sin \left(\pi^{n} J_{C H} \Delta\right) \\
\mathrm{TQ} \quad \mathrm{SQ} \\
\mathrm{TQ}
\end{gathered}
$$

\section{CHH'H' spin system}


$H_{Z}^{r}$

$\stackrel{(90)^{\circ} x H}{\longrightarrow} \stackrel{\pi^{n} J_{C H} \Delta 2 H_{Z} C_{Z}}{\longrightarrow} \stackrel{\pi^{n} J_{H H^{\prime}} \Delta 2 H_{Z} H^{\prime}{ }_{Z} \pi^{n} J_{H H^{\prime \prime}} \Delta 2 H_{Z} H^{\prime \prime} Z}{\longrightarrow} \stackrel{(180)^{\circ} x H}{\longrightarrow} \stackrel{(180)^{\circ} x H^{\prime}(180)^{\circ} x H^{\prime \prime}(180)^{\circ} x C}{\longrightarrow} \stackrel{(90)^{\circ} y H(90)^{\circ} y H^{\prime}(90)^{\circ} y H^{\prime \prime}(!}{\longrightarrow} \stackrel{\longrightarrow}{\longrightarrow} \stackrel{\longrightarrow}{\longrightarrow}$

$$
-2 H_{Z}^{r} C_{Y} \cos \left(\pi J_{H H^{\prime}} \Delta\right) \cos \left(\pi J_{H H^{\prime \prime}} \Delta\right) \sin \left(\pi^{n} J_{C H} \Delta\right)
$$$$
+4 H_{Y}^{r} H_{X}^{\prime \prime} C_{Y} \cos \left(\pi J_{H H^{\prime}} \Delta\right) \sin \left(\pi J_{H H^{\prime \prime}} \Delta\right) \sin \left(\pi^{n} J_{C H} \Delta\right)
$$$$
+4 H_{Y}^{r} H_{X}^{\prime} C_{Y} \sin \left(\pi J_{H H^{\prime}} \Delta\right) \cos \left(\pi J_{H H^{\prime \prime}} \Delta\right) \sin \left(\pi^{n} J_{C H} \Delta\right)
$$$$
+8 H_{Z}^{r} H_{X}^{\prime} H_{X}^{\prime \prime} C_{Y} \sin \left(\pi J_{H H^{\prime}} \Delta\right) \sin \left(\pi J_{H H^{\prime \prime}} \Delta\right) \sin \left(\pi^{n} J_{C H} \Delta\right)
$$

The single quantum carbon coherence $2 \mathrm{H}_{Z}{ }_{Z} \mathrm{C}_{Y}$ is transformed into $S Q$ coherences as described above and the $4 H^{r}{ }_{Y} H_{X}^{\prime} C_{Y}, 4 H^{r} H^{\prime \prime}{ }_{X} C_{Y}$ and $8 H^{r} H^{\prime} x H^{\prime \prime} C_{Y}$ coherences term are correspondingly transformed into a sum of TQ and SQ quantum coherences (the trigonometric factors have been omitted for clarity):

$$
\begin{aligned}
& -2 H_{Z}^{r} C_{Y} \rightarrow i H_{Z}^{r}\left(C_{+}-C_{-}\right) \\
& \text {SQ } \\
& +4 H_{Y}^{r} H_{X}^{\prime} C_{Y} \rightarrow \\
& -0.5\left(H_{+}^{r} H_{+}^{\prime}-H_{+}^{r} H_{-}^{\prime}+H_{-}^{r} H_{+}^{\prime}+H_{-}^{r} H_{-}^{\prime}\right)\left(C_{+}-C_{-}\right) \\
& \begin{array}{llll}
T Q & S Q & S Q & T Q
\end{array} \\
& +4 H_{Y}^{r} H_{X}^{\prime \prime} C_{Y} \rightarrow \\
& -0.5\left(H_{+}^{r} H_{+}^{\prime \prime}-H_{+}^{r} H_{-}^{\prime \prime}+H_{-}^{r} H_{+}^{\prime \prime}+H_{-}^{r} H_{-}^{\prime \prime}\right)\left(C_{+}-C_{-}\right) \\
& \begin{array}{llll}
\mathrm{TQ} & \mathrm{SQ} & \mathrm{SQ} & \mathrm{TQ}
\end{array} \\
& +8 H_{Z}^{r} H_{X}^{\prime} H_{X}^{\prime \prime} C_{Y} \rightarrow \\
& -i\left(H_{+}^{\prime} H_{+}^{\prime \prime}+H_{+}^{\prime} H_{-}^{\prime \prime}+H_{-}^{\prime} H_{+}^{\prime \prime}+H_{-}^{\prime} H_{-}^{\prime \prime}\right)\left(C_{+}-C_{-}\right) H_{Z}^{r}
\end{aligned}
$$

\section{$C(H)_{n}, n>3$ spin systems}


The product operator analysis for a $\mathrm{C}(\mathrm{H}) n, n>3$-spin system becomes very lengthy, but some general features can still be derived. For a five-spin system CHH'H'H'", after the INEPT transfer, the following eight operators are present: $2 \mathrm{H}_{Z}{ }_{Z} \mathrm{C}_{Y}$

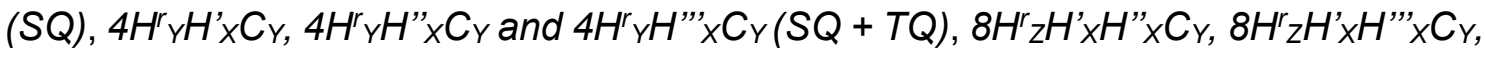
and $8 H^{r} H^{\prime}{ }^{\prime \prime} H^{\prime \prime \prime} \times C_{Y}(S Q+T Q)$, and finally $16 H^{r}{ }^{\prime} H^{\prime} x H^{\prime \prime} \times H^{\prime \prime \prime} \times C_{Y}(S Q+T Q+Q Q)$. The respective $\mathrm{TQ}$ coherences will be finally converted into observable proton single quantum coherences.

\section{Stage $b$}

\section{The selection of coherence pathways by magnetic field gradients}

The coherences present at stage a continue to evolve in $\mathrm{t}_{1}$ exclusively under the influence of carbon chemical shifts and the effect of the second ${ }^{13} \mathrm{C} 180^{\circ}$ pulse. For understanding the effect of the magnetic field gradient $\mathrm{G} 1$ (in combination with gradient G2) note the following:

The various coherences that are created during the HTQC experiment correspond to different nuclei specific coherence levels $C L_{k}$. As an example the coherence $\mathrm{H}^{r}{ }_{+} H^{\prime}{ }_{+} \mathrm{C}_{+}$has coherence levels $+2\left({ }^{1} \mathrm{H}\right),+1\left({ }^{13} \mathrm{C}\right)$, i.e. represents triple quantum coherence and $H^{r}+H^{\prime}-C_{+}$has coherence levels $0\left({ }^{1} H\right),+1\left({ }^{13} \mathrm{C}\right)$, i.e. represents single quantum ${ }^{13} \mathrm{C}$ coherence.

For selecting specific coherence pathways these levels but also the corresponding magnetogyric ratios of the involved nuclei have to be considered. Since with the proposed LR-HTQC experiment the finally detected ${ }^{1} \mathrm{H}$ single quantum coherence (at stage $\mathrm{c}$ ), should originate exclusively from intermediate triple quantum (TQ) coherences at stage b, their further evolution in the residual part of the pulse sequence and in particular the coherence level of the finally detected ${ }^{1} \mathrm{H}$ coherence must be evaluated and the condition below must be fulfilled.

In order to re-phase final ${ }^{1} \mathrm{H}$ coherence originating from any of these $T Q$ terms, de-phased by the first gradient $\mathrm{G} 1$, the condition for the individual gradient strengths $G_{i}$ and the correspondingly affected (gyromagnetic-weighted) coherence levels $C L_{k}$ across the pulse sequence is as follows:[35-37] 


$$
\Sigma_{i} G_{i} \cdot C L_{i} \cdot \gamma_{i}=0
$$

Anticipating the effect of the two magnetic field gradients G1 and G2 (see below), note that with the corresponding gradient strength settings (4:9 or 4:7) the SQ coherences, if finally transformed into ${ }^{1} \mathrm{H}$ coherences, will remain dephased and will not be detectable.

On the other hand with any of the gradient strength settings, depending on whether the individual TQ terms develop into final ${ }^{1} \mathrm{H}$ coherence at ${ }^{1} \mathrm{H}$ coherence level -1 or +1 and depending on which of these two levels will be detected by the $F_{2-}$ quadrature detection, only one of the TQ coherences $H^{r}+H^{\prime}{ }_{+} C_{+}\left(\mathrm{CL}_{i \gamma}=+9\right), H^{r}-H_{-}^{\prime} C_{+}$ $\left(C_{i \gamma}=-7\right), H^{r}-H_{-}^{\prime} C_{-}\left(C_{i \gamma}=-9\right)$ or $H^{r}+H^{\prime}+C_{-}\left(C_{i \gamma}=+7\right)$ will survive and will be transferred into a final ${ }^{1} \mathrm{H}$ signal $\left(\mathrm{CL}_{i \gamma}=-4\right)$.

With e.g. the gradient strengths set to $4: 9$ and with $F_{2}$-quadrature detection at ${ }^{1} \mathrm{H}$ coherence level -1 , it is the TQ-term $\mathrm{H}^{r}{ }_{+} \mathrm{H}_{+} \mathrm{C}_{+}-$transferred in the residual part of the pulse sequence into final ${ }^{1} \mathrm{H}$ coherence at coherence level -1 - which will be detected.

\section{CHH' spin system}

$$
0.5\left(H_{-}^{r} H_{-}^{\prime}\right)\left(C_{-}\right) \stackrel{t_{1} / 2-(180)^{\circ} x H^{r},(180)^{\circ} x H^{\prime}-t_{1} / 2-(180)^{\circ} x C}{\longrightarrow} 0.5\left(H_{+}^{r} H_{+}^{\prime}\right) C_{+} e^{i \pi \Omega_{C} t_{1}}
$$

\section{CHH'H" spin system}

$$
\begin{gathered}
0.5\left(H_{-}^{r} H_{-}^{\prime}\right) C_{-} \stackrel{t_{1} / 2-(180)^{\circ} x H^{r},(180)^{\circ} x H^{\prime}-t_{1} / 2-}{\longrightarrow} \stackrel{(180)^{\circ} x C}{\longrightarrow} 0.5\left(H_{+}^{r} H_{+}^{\prime}\right) C_{+} e^{i \pi \Omega_{C} t_{1}} \\
0.5\left(H_{-}^{r} H_{-}^{\prime \prime}\right) C_{-} \stackrel{t_{1} / 2-(180)^{\circ} x H^{r},(180)^{\circ} x H^{\prime \prime}-t_{1} / 2-}{\longrightarrow} \stackrel{(180)^{\circ} x C}{\longrightarrow} 0.5\left(H_{+}^{r} H_{+}^{\prime \prime}\right) C_{+} e^{i \pi \Omega_{C} t_{1}} \\
i\left(H_{-}^{\prime} H_{-}^{\prime \prime}\right) C_{-} H_{Z}^{r} \\
\stackrel{t_{1} / 2-(180)^{\circ} x H^{r},(180)^{\circ} x H^{\prime},(180)^{\circ} x H^{\prime \prime}-t_{1} / 2-}{\longrightarrow} \stackrel{(180)^{\circ} x C}{\longrightarrow} i\left(H_{+}^{\prime} H_{+}^{\prime \prime}\right) C_{+} H_{Z}^{r} e^{i \pi \Omega_{C} t_{1}}
\end{gathered}
$$




\section{Stage c:}

\section{CHH' spin system}

$$
0.5\left(H_{+}^{r} H_{+}^{\prime}\right) C_{+} e^{i \pi \Omega_{C} t_{1}} \stackrel{\left(90^{\circ}\right)_{x} C\left(90^{\circ}\right)_{x} H^{r}\left(90^{\circ}\right)_{x} H^{\prime} G_{1}+G_{2}}{\longrightarrow}-0.25 H_{-}^{r} H_{Z}^{\prime} C_{Z} e^{i \pi \Omega_{C} t_{1}}
$$

This observable coherence $H^{r} \cdot H_{z}{ }^{\prime} C_{z}$ represents absorptive $x$-magnetization of spin $H^{r}$ doubly antiphase with respect to spin $\mathrm{H}^{\prime}$ and $\mathrm{C}$ and enables also quadrature detection in ${ }^{13} \mathrm{C}$-frequency domain $\mathrm{F}_{1}$.

Note at this stage and most important, that the presence of cross-peaks with such mixed phases can lead to an accidental signal cancellation, especially if the coupling constants ${ }^{n} J_{\mathrm{CH}}$ and $\mathrm{JHH}$ and the linewidths of the individual lines are of similar or the same size. In this context an adequate digital resolution in $F_{2}$, i.e. data recorded with sufficient points is mandatory. ${ }^{[38]}$

\section{CHH'H" spin system}

$$
\begin{gathered}
0.5\left(H_{+}^{r} H_{+}^{\prime}\right) C_{+} e^{i \pi \Omega_{C} t_{1}} \stackrel{(90)^{\circ} x C}{\longrightarrow} \stackrel{(90)^{\circ} x H^{r}}{\longrightarrow} \stackrel{(90)^{\circ} x H^{\prime}}{\longrightarrow} \stackrel{(90)^{\circ} x H^{\prime \prime}}{\longrightarrow} \stackrel{G_{1}+G_{2}}{\longrightarrow}-0.25 H_{-}^{r} H_{Z}^{\prime} C_{Z} e^{i \pi \Omega_{C} t_{1}} \\
0.5\left(H_{+}^{r} H_{+}^{\prime \prime}\right) C_{+} e^{i \pi \Omega_{C} t_{1}} \stackrel{(90)^{\circ} x C(90)^{\circ} x H^{r}(90)^{\circ} x H^{\prime}}{\longrightarrow} \stackrel{(90)^{\circ} x H^{\prime \prime}}{\longrightarrow} \stackrel{G_{1}+G_{2}}{\longrightarrow}-0.25 H_{-}^{r} H_{Z}^{\prime \prime} C_{Z} e^{i \pi \Omega_{C} t_{1}} \\
i\left(H_{+}^{\prime} H_{+}^{\prime \prime}\right) C_{+} H_{Z}^{r} e^{i \pi \Omega_{C} t_{1}} \stackrel{(90)^{\circ} x C(90)^{\circ} x H^{r}}{\longrightarrow} \stackrel{(90)^{\circ} x H^{\prime}}{\longrightarrow} \stackrel{(90)^{\circ} x H^{\prime \prime}}{\longrightarrow} \stackrel{G_{1}+G_{2}}{\longrightarrow}-0.5 i H_{-}^{r} H_{Z}^{\prime} H_{Z}^{\prime \prime} C_{Z} e^{i \pi \Omega_{C} t_{1}}
\end{gathered}
$$

The term $H^{r}-H^{\prime} z C_{z}$ represents $x$-magnetization of spin $\mathrm{H}^{r}$ which is doubly antiphase with respect to spin $C$ and spin $H^{\prime}$, the term $H^{r}-H^{\prime \prime}{ }_{z} C_{z}$ represents $x$ magnetization of spin $\mathrm{H}^{r}$ which is doubly antiphase with respect to spin $\mathrm{C}$ and spin $\mathrm{H}^{\prime \prime}$, 
and the term $i H^{r}-H_{z}^{\prime} H^{\prime \prime} z C_{z}$ represents $y$-magnetization of spin $\mathrm{H}^{r}$ which is triply antiphase with respect to spin $\mathrm{C}$ and spins H' and H'.

At this stage it's useful to compare the observable coherences present before acquisition as obtained with the LR-HSQC and LR-HTQC experiments:

\section{CH spin systems}

LR-HSQC

$$
-0.5 i H_{-}^{r} C_{Z} e^{i \pi \Omega_{C} t_{1}} \sin \left(\pi^{n} J_{C H} \Delta\right)
$$

\section{LR-HTQC}

No signals

Whereas with the LR-HSQC experiment a cross peak is visible, with the LRHTQC experiment, the cross peaks of $\mathrm{CH}$ two-spin systems (with no additional $\mathrm{H}, \mathrm{H}$ couplings) vanish as intended (Figure S2).

\section{CHH' spin systems}

LR-HSQC

$$
\begin{aligned}
& -0.5 i H_{-}^{r} C_{Z} e^{i \pi \Omega_{C} t_{1}} \cos \left(\pi J_{H H^{\prime}} \Delta\right) \sin \left(\pi^{n} J_{C H} \Delta\right) \\
& +0.5 H_{-}^{r} H_{Z}^{\prime} C_{Z} e^{i \pi \Omega_{C} t_{1}} \sin \left(\pi J_{H H^{\prime}} \Delta\right) \sin \left(\pi^{n} J_{C H} \Delta\right)
\end{aligned}
$$

\section{LR-HTQC}

$$
-0.25 H_{-}^{r} H_{Z}^{\prime} C_{Z} e^{i \pi \Omega_{C} t_{1}} \sin \left(\pi J_{H H^{\prime}} \Delta\right) \sin \left(\pi^{n} J_{C H} \Delta\right)
$$

With the LR-HTQC experiment one single term contributes to the cross peak, whereas with the LR-HSQC experiment two terms contribute. Thus, in LR-HTQC spectra the intensities of cross peaks of $\mathrm{CHH}^{\prime}$ three spin systems are significantly reduced. However, the extent of intensity reduction is difficult to be estimated 
beforehand, since it depends on the long-range evolution delay $\Delta$, the heteronuclear ${ }^{n} J_{\mathrm{CH}}$ and the homonuclear $\mathrm{JHH}_{\mathrm{H}}$ coupling constants.

It appears that real doublets in the ${ }^{1} \mathrm{H}$ NMR spectrum, as for methyl groups in $(\mathrm{Me})_{2} \mathrm{CH}$ or $\mathrm{MeCH}$ - fragments, or aromatic protons, are canceled or at least be strongly reduced in LR-HTQC spectra by tuning the long-range evolution delay $\Delta$ to $1 / J_{H H^{\prime}}$ the (Figure S3).

\section{CHH'H" spin systems}

\section{LR-HSQC}

$$
\begin{aligned}
& -0.5 H_{-}^{r} C_{Z} e^{i \pi \Omega_{C} t_{1}} \cos \left(\pi J_{H H^{\prime}} \Delta\right) \cos \left(\pi J_{H H^{\prime \prime}} \Delta\right) \sin \left(\pi^{n} J_{C H} \Delta\right) \\
- & 0.5 i H_{-}^{r} H_{Z}^{\prime} C_{Z} e^{i \pi \Omega_{C} t_{1}} \sin \left(\pi J_{H H^{\prime}} \Delta\right) \cos \left(\pi J_{H H^{\prime \prime}} \Delta\right) \sin \left(\pi^{n} J_{C H} \Delta\right) \\
- & 0.5 i H_{-}^{r} H_{Z}^{\prime \prime} C_{Z} e^{i \pi \Omega_{C} t_{1}} \cos \left(\pi J_{H H^{\prime}} \Delta\right) \sin \left(\pi J_{H H^{\prime \prime}} \Delta\right) \sin \left(\pi^{n} J_{C H} \Delta\right) \\
+ & H_{-}^{r} H_{Z}^{\prime} H_{Z}^{\prime \prime} C_{Z} e^{i \pi \Omega_{C} t_{1}} \sin \left(\pi J_{H H^{\prime}} \Delta\right) \sin \left(\pi J_{H H^{\prime \prime}} \Delta\right) \sin \left(\pi^{n} J_{C H} \Delta\right)
\end{aligned}
$$

\section{LR-HTQC}

$$
\begin{aligned}
& -0.25 H_{-}^{r} H_{Z}^{\prime} C_{Z} e^{i \pi \Omega_{C} t_{1}} \sin \left(\pi J_{H H^{\prime}} \Delta\right) \cos \left(\pi J_{H H^{\prime \prime}} \Delta\right) \sin \left(\pi^{n} J_{C H} \Delta\right) \\
& -0.25 H_{-}^{r} H_{Z}^{\prime \prime} C_{Z} e^{i \pi \Omega_{C} t_{1}} \cos \left(\pi J_{H H^{\prime}} \Delta\right) \sin \left(\pi J_{H H^{\prime \prime}} \Delta\right) \sin \left(\pi^{n} J_{C H} \Delta\right) \\
& -0.5 i H_{-}^{r} H_{Z}^{\prime} H_{Z}^{\prime \prime} C_{Z} e^{i \pi \Omega_{C} t_{1}} \sin \left(\pi J_{H H^{\prime}} \Delta\right) \sin \left(\pi J_{H H^{\prime \prime}} \Delta\right) \sin \left(\pi^{n} J_{C H} \Delta\right)
\end{aligned}
$$

With the LR-HTQC experiment, the intensity of cross peaks of CHH'H" spin systems are reduced compared to the LR-HSQC experiment, but to a lesser extent. As mentioned for $\mathrm{CHH}^{\prime}$ spin systems, predicting the loss of intensity beforehand is difficult since it depends on the long-range evolution delay $\Delta$, the heteronuclear ${ }^{n} \mathrm{~J}_{\mathrm{CH}}-$ and both homonuclear $\mathrm{JHH}$ - and $\mathrm{JHH}_{\mathrm{H}}$ ' coupling constants. Yet, in general, for standard $\Delta$ values $\left(50<\Delta<70 \mathrm{~ms}\right.$ ) and with both proton-proton couplings $\mathrm{JHH}^{\prime}$ and $\mathrm{JHH}^{\prime \prime}<3 \mathrm{~Hz}$, the respective cross peak of a CHH'H" spin system will be weak in the LR-HTQC spectrum $\left(\cos \left(\pi J_{H^{\prime}} \Delta\right) \rightarrow 1\right.$ and $\sin \left(\pi J_{H H^{\prime}} \Delta\right) \rightarrow 0$ ) compared to the same cross peak in the LR-HSQC spectrum (Figure S6). On the other hand, if both proton-proton couplings $J_{H H^{\prime}}$ and $J_{H H}$ " are $>5 \mathrm{~Hz}$, the intensities of the same cross peak in the LR-HTQC 
spectrum and in the LR-HSQC spectrum will be almost the same $\left(\cos \left(\pi J_{H H} \Delta\right) \rightarrow 0\right.$ and $\sin \left(\pi J_{H H^{\prime}} \Delta\right) \rightarrow 1$ ) (Figure S7).

Thus, and compared to the LR-HSQC experiment, the LR-HTQC experiment may be advantageous to suppress the often intense long-range responses (and the associated residual one-bond responses surviving the low-pass $J$ filter step if added) of $\mathrm{CH}$ spin pairs (singlets in the ${ }^{1} \mathrm{H}$ NMR spectrum). Furthermore, with the LR-HTQC experiment the intensity of the cross peaks of $\mathrm{CHH}^{\prime}$ spin systems (doublets in the ${ }^{1} \mathrm{H}$ NMR spectrum) may be significantly reduced or even suppressed. Overall this simplifies the appearance of heteronuclear long-range shift correlation spectra, a feature which can be very useful particularly with crowded spectra, as shown in the experimental results section.

\section{Experimental Results \\ Cholesteryl acetate}

In Figure 3, sections of 2D LR-HSQC and LR-HTQC spectra of $100 \mathrm{mmol}$ cholesteryl acetate (Figure 2) dissolved in $\mathrm{CDCl}_{3}$ showing long-range correlations in the aliphatic region are presented. With the initial delay $\Delta$ adjusted to $62.5 \mathrm{~ms}$, the differences in intensity in the LR-HSQC and LR-HTQC spectra are expected to be most pronounced and desirable for $\mathrm{CH}$ groups with no visible additional $\mathrm{J}_{\mathrm{HH}}$ couplings, i.e. $\mathrm{CH}_{3}(1)$ at $\delta=2.02 \mathrm{ppm}, \mathrm{CH}_{3}(9)$ at $\delta=1.01 \mathrm{ppm}$ and $\mathrm{CH}_{3}(19)$ at $\delta=0.67 \mathrm{ppm}$, that all appear as singlets in the ${ }^{1} \mathrm{H}$ NMR spectrum (Figure S8). 


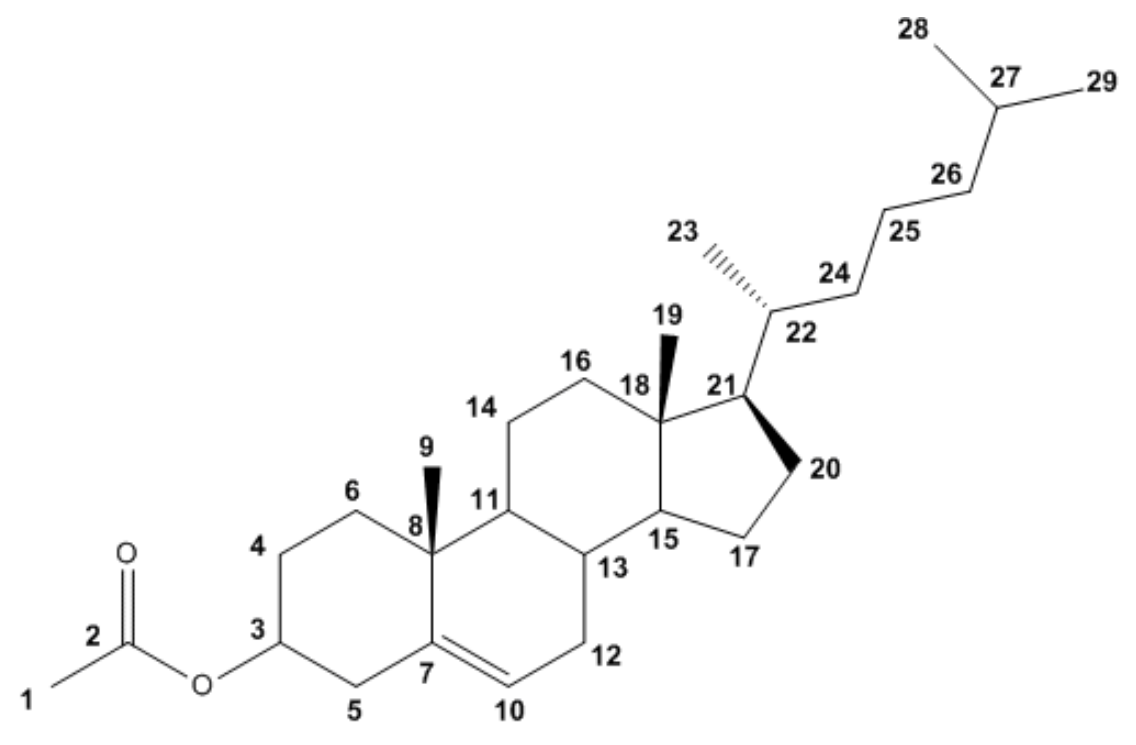

Figure 2. Chemical structure of cholesteryl acetate and atom numbering used in the text. 


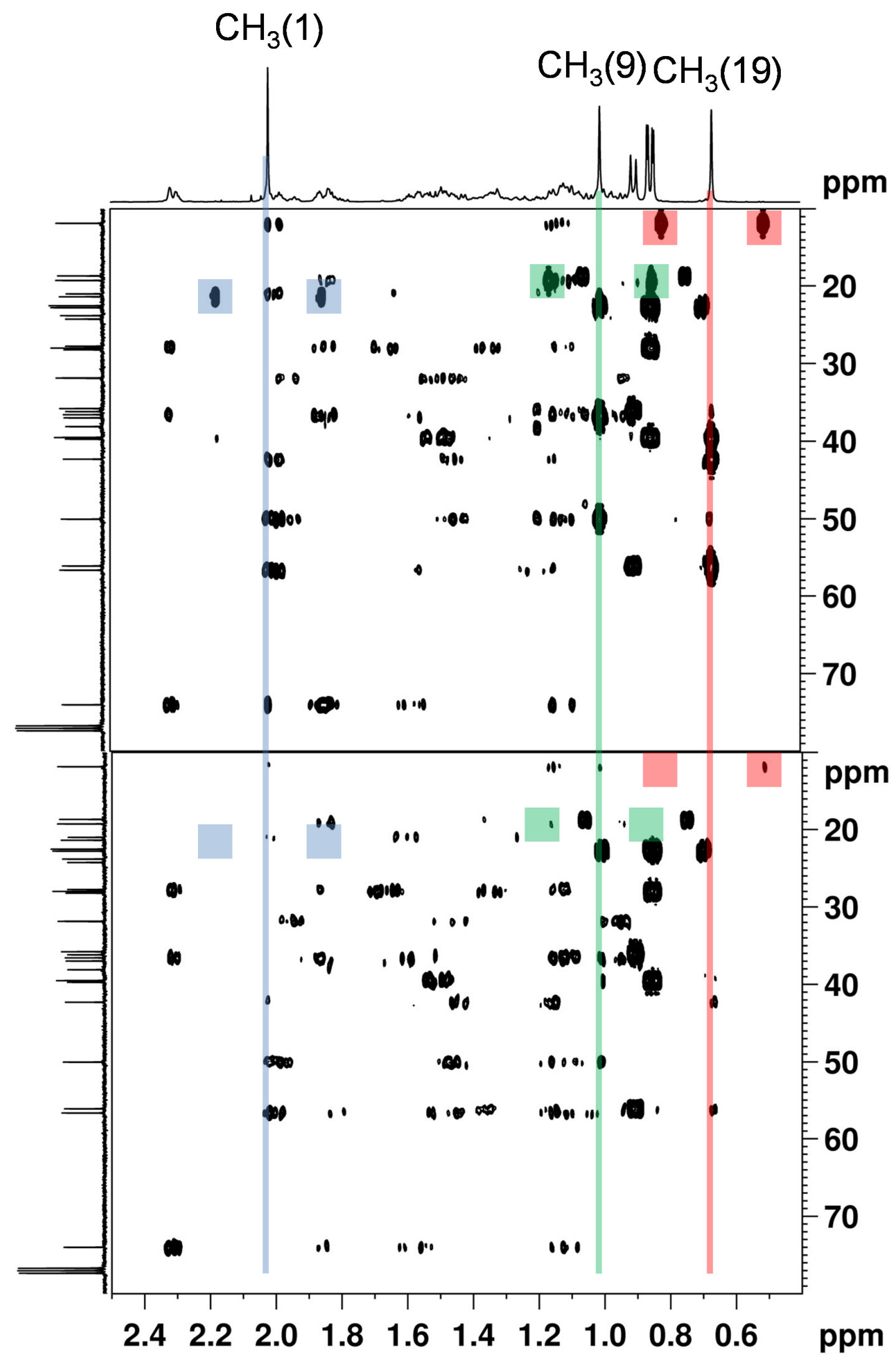


Figure 3. Segments of the $8 \mathrm{~Hz}$ adjusted LR-HSQC (top) and LR-HTQC (bottom) spectra of $100 \mathrm{mmol}$ cholesteryl acetate dissolved in $\mathrm{CDCl}_{3}$ showing ${ }^{n} J_{C H}$ long-range correlations of the aliphatic resonances. Both spectra are displayed at the same noise level.

The (singlet) resonances of $\mathrm{CH}_{3}(1)$ at $\delta=2.02 \mathrm{ppm}, \mathrm{CH}_{3}(9)$ at $\delta=1.01 \mathrm{ppm}$ and $\mathrm{CH}_{3}(19)$ at $\delta=0.67 \mathrm{ppm}$ (Figure 5) exemplify the case of $\mathrm{CH}$ spin systems, while the (doublet) resonances of $\mathrm{CH}_{3}(23)$ at $\delta=0.91 \mathrm{ppm}$ and $\mathrm{CH}_{3}(28,29)$ at $\delta=0.86$ and 0.865 ppm illustrate the case of $\mathrm{CHH}^{\prime}$ spin systems $\left({ }^{3} \mathrm{~J}_{\mathrm{H} 23 \mathrm{H} 22}=6.7 \mathrm{~Hz},{ }^{3} \mathrm{~J}_{\mathrm{H} 28 \mathrm{H} 27}=6.6 \mathrm{~Hz}\right)$. Clearly, as predicted, the long-range responses and the associated one-bond responses of the methyl groups 1 and 9 are completely suppressed or, for the $\mathrm{CH}_{3}(19)$ resonance at $\delta=0.67 \mathrm{ppm}$, at least significantly reduced.

The $\mathrm{CH}_{3}(9)$ resonance at $\delta=1.01 \mathrm{ppm}$ clearly exemplifies the potential advantages with TQ-filtering that can be achieved with this new LR-HTQC experiment compared to the LR-HSQC experiment. The HSQC spectrum (Figure S9) reveals four protons resonating at $\delta=1.01 \pm 0.03 \mathrm{ppm}: \mathrm{CH}_{3}(9), \mathrm{H} 15, \mathrm{H} 17 \mathrm{a}$, and $\mathrm{H} 24 \mathrm{a}$ (Table S1). In the LR-HSQC spectrum (Figure 3) two very strong correlations within this narrow ${ }^{1} \mathrm{H}$ shift range with carbon $\mathrm{C} 8$ at $\delta=36.6 \mathrm{ppm}\left({ }^{2} J_{\mathrm{H} 9 \mathrm{C} 8}\right)$ and with carbon $\mathrm{C} 11$ at $\delta=50.0$ ppm ( $\left.{ }^{3} J_{\mathrm{H} 9 \mathrm{C} 11}\right)$ dominate and potentially mask other weak correlations arising either from $\mathrm{H} 15, \mathrm{H} 17 \mathrm{a}$, or $\mathrm{H} 24 \mathrm{a}$. For instance, the weak cross peak at $\delta=1.01 \mathrm{ppm} / \delta=50.0$ visible in the LR-HTQC spectrum, ascribed to the ${ }^{3} \mathrm{~J}_{\mathrm{CH}}$ long-range correlation between H15 and C11.

Interestingly, the long-range responses of the methyl group $\mathrm{CH}_{3}(19)$ at $\delta=0.67$ ppm are not completely suppressed in the LR-HTQC spectrum. This suggests that the proton resonance of $\mathrm{CH}_{3}(19)$ is actually a multiplet instead of a singlet, because of very weak ${ }^{4} \mathrm{JHH}$ long range couplings with $\mathrm{H} 15, \mathrm{H} 16 \mathrm{a} / \mathrm{H} 16 \mathrm{~b}$ and $\mathrm{H} 21$, which is proved by a COSY spectrum showing the corresponding very weak cross peaks between $\mathrm{CH}_{3}(19)$ and $\mathrm{H} 15, \mathrm{H} 16 \mathrm{a}$, and $\mathrm{H} 21$.

Consequently, the ${ }^{1} \mathrm{H}$ resonance of $\mathrm{CH}_{3}(19)$ must be considered as part of a $\mathrm{C}(\mathrm{H}) \mathrm{n}$ spin system. A short analysis based e.g. on equation 16 shows that with very small J JH' coupling constants and because the cumulative effect of the various $\sin \left(\pi J_{H} H^{\prime} \Delta\right)$ terms the intensity of the associated cross peaks weaken close to zero.

On the other hand, the very intense long-range responses and the associated one-bond responses of $\mathrm{CH}_{3}(23)$ at $\delta=0.91 \mathrm{ppm}$ and of $\mathrm{CH}_{3}(28,29)$ at $\delta=0.86$ and 
0.865 ppm, with doublets in the ${ }^{1} \mathrm{H}$ spectrum, are clearly visible in the LR-HTQC spectrum (Figure 3) and only slightly less intense than in the LR-HSQC spectrum.

The residual cross peaks in the LR-HTQC spectrum are equally or less intense compared to the corresponding cross peaks in the LR-HSQC spectrum (Figures 4, 5). As outlined before the decrease in intensity for these cross peaks is difficult to predict, and depends on both the number and the magnitude of the homonuclear proton-proton couplings $J_{\mathrm{HH}^{\prime}}$ (magnitude of the various $\cos \left(\pi J_{\mathrm{HH}} \Delta\right)$ and $\sin \left(\pi J_{\mathrm{HH}} \Delta\right)$ terms).

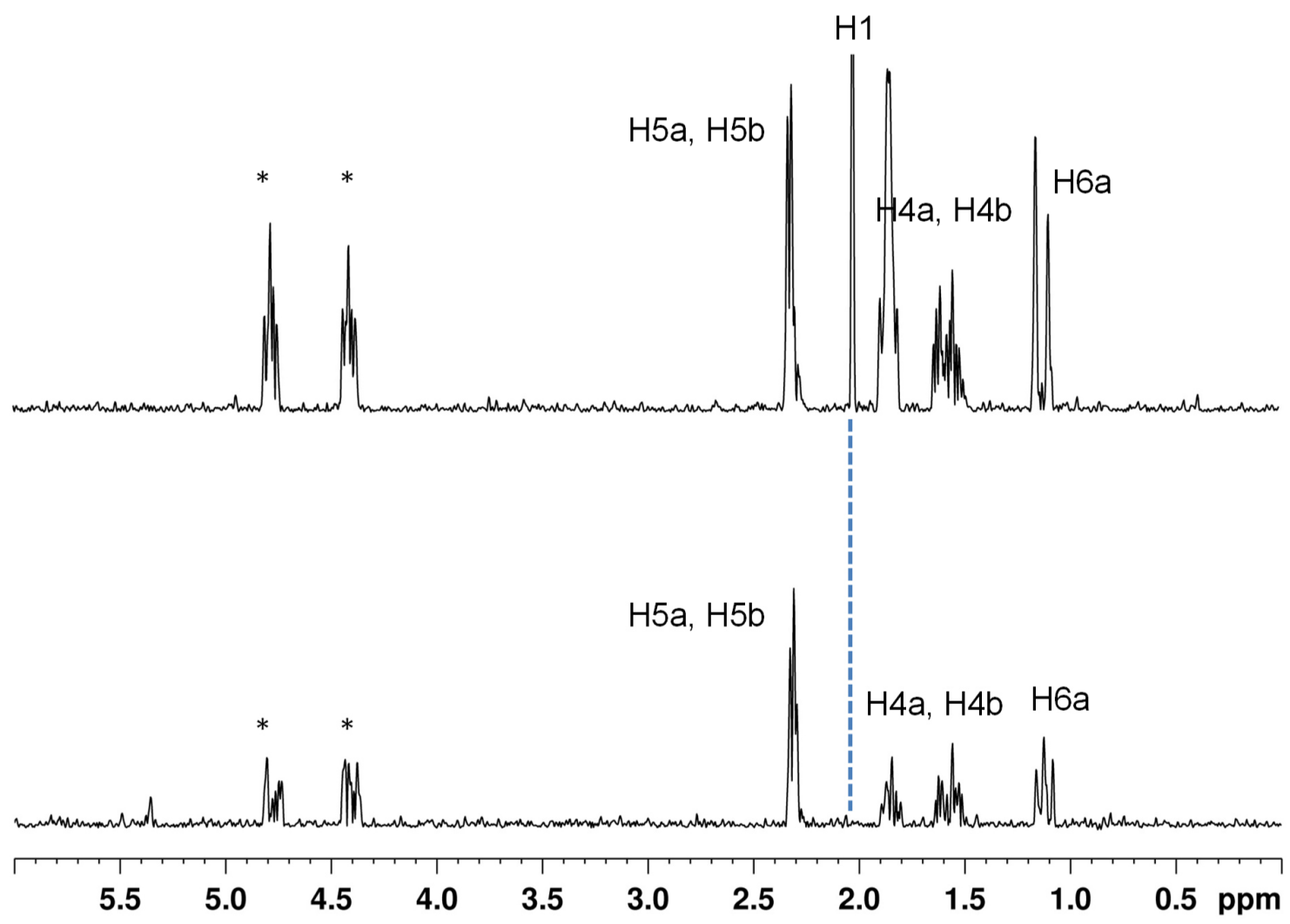

Figure 4. 1D rows extracted from the $7 \mathrm{~Hz}$ optimized LR-HTQC (bottom) and LR$\mathrm{HSQC}$ (top) spectra of cholesteryl acetate dissolved in $\mathrm{CDCl}_{3}$ showing the long-range correlations associated with the $\mathrm{C} 3$ resonance $(\delta=74.0 \mathrm{ppm})$. Both spectra are displayed with the same noise level. ${ }^{1} \mathrm{~J}_{\mathrm{CH}}$ artifacts are marked with asterisk. 


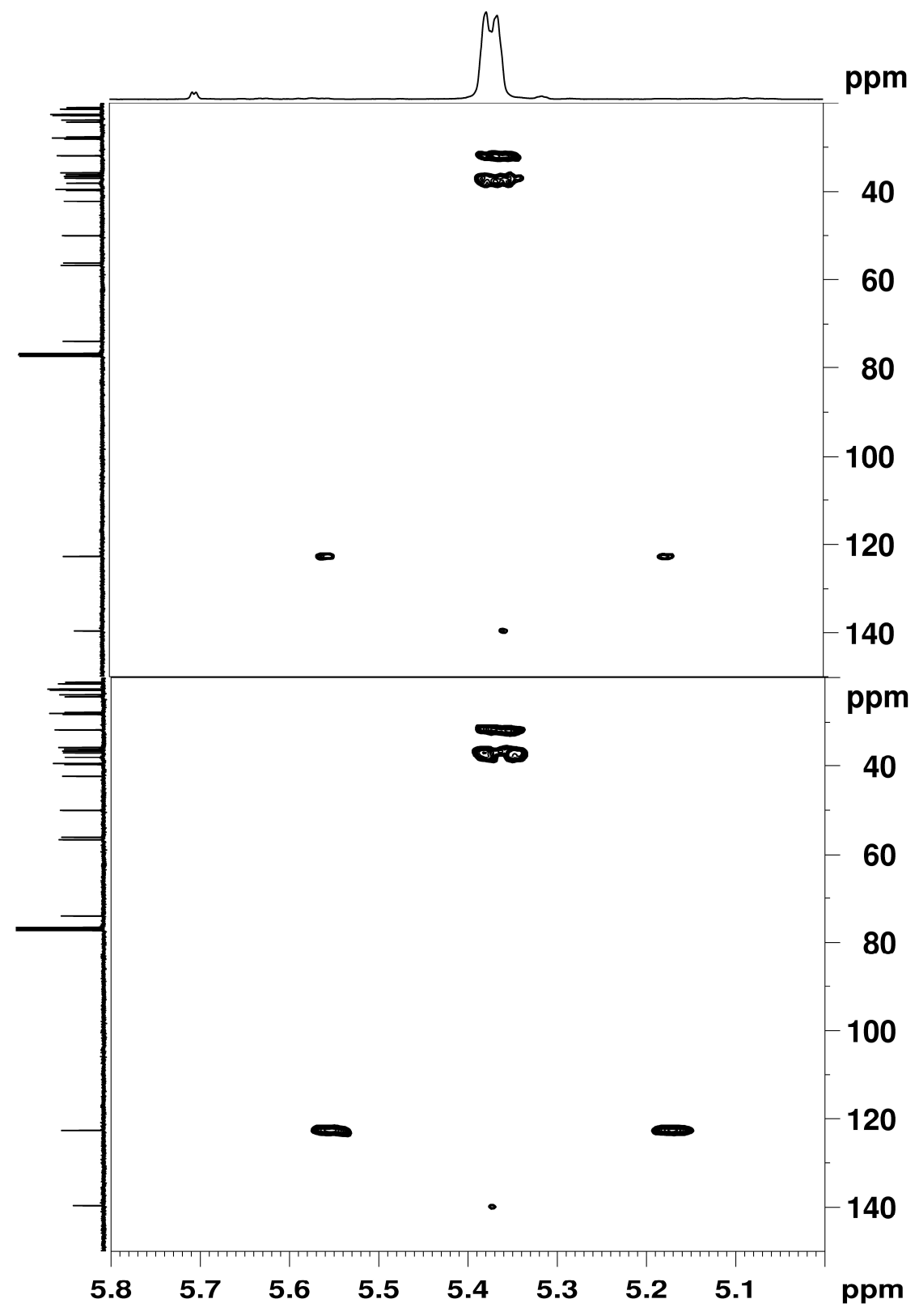

Figure 5. Segments of the $7 \mathrm{~Hz}$ adjusted LR-HSQC (bottom) and LR-HTQC (top) spectra of $100 \mathrm{mmol}$ cholesteryl acetate dissolved in $\mathrm{CDCl}_{3}$ showing ${ }^{n} \mathrm{~J}_{\mathrm{CH}}$ long-range correlations of $\mathrm{H} 10$. Both spectra are displayed at the same noise level. 


\section{Strychnine}

LR-HSQC and LR-HTQC spectra for a standard strychnine sample dissolved in $\mathrm{CDCl}_{3}$ are provided in the Supplementary Information (Figure S10). Of note, as strychnine does not possess an isolated $\mathrm{CH}$ spin pair, only the general reduction of the cross peak's intensity in the LR-HTQC is apparent as compared with the LR-HSQC spectrum. As aforementioned, the extent of intensity reduction is difficult to be estimated beforehand, since it depends on the long-range evolution delay $\Delta$, the heteronuclear ${ }^{n} \mathrm{~J}_{\mathrm{CH}}$ and the homonuclear $\mathrm{J}_{\mathrm{HH}}$ ' coupling constants. With strychnine, it can be seen that the cross peak's intensity is significantly reduced in the aliphatic region (Figure S11), while it is much less reduced for the aromatic positions 1-4 and for the carbonyl group 10. (Figure S12)

\section{Conclusion}

LR-HSQC spectra and more generally experiments aimed at obtaining longrange heteronuclear shift correlation spectra often provide an abundance of cross peaks and a density of information too high to be used directly without "filtering out" shorter range correlations. Furthermore, significant differences in intensity between cross peaks exist, which may hinder the visualization of weak, often crucial cross peaks.

Facing these problems and inspired by related heteronuclear shift correlation pulse sequences, we have developed the LR-HTQC experiment, which provides an easier way to evaluate long-range heteronuclear shift correlation. By selecting specific coherence pathways with intermediate triple quantum coherences, the LR-HTQC experiment not only removes the intense cross peaks of $\mathrm{CH}$ spin pairs, but compensates for strong differences in intensity among the residual cross peaks. The intensity of cross peaks originating from $\mathrm{CHH}^{\prime}$ spin systems is substantially reduced, while the intensity of cross peaks of $\mathrm{CHH}^{\prime} \mathrm{H}^{\prime \prime}$ and $\mathrm{C}(\mathrm{H})_{n}, n>3$ spin systems is less affected.

However, the sensitivity of the LR-HTQC experiment is lower compared to LRHSQC, which may preclude its use for low concentration samples. Additionally, as shown with the model compounds cholesteryl acetate and strychnine in this study, the 
LR-HTQC experiment was not designed to substitute the LR-HSQC or LR-HSQMBC experiments, but should be seen as a complementary method to those experiments, which should remain the first experiment to be acquired for detecting long.-range correlations. The LR-HTQC experiment may be advantageously used in the case of severe overlap in the LR-HSQC spectra for easier access to the relevant structural information.

\section{Experimental Section}

The product operator equations were hand calculated and cross-checked using the Software SIM3 (R.E.D McClung \& T. T. Nakashima, 1991, "Computer Simulation of Multiple-Pulse and Two-Dimensional FT NMR Experiments using Product Operators in the Spherical Basis", Department of Chemistry, University of Alberta, Canada).

The simulations shown in the Supplementary information have been performed with the BRUKER NMRSIM program for Windows (version 6.0.3. 2019).

All NMR experiments were recorded on a BRUKER AvancelllHD-400 spectrometer equipped with a dual-channel $5 \mathrm{~mm}$ SmartProbe ${ }^{\circledR}$ incorporating a zgradient coil. The test samples were strychnine $(30 \mathrm{mg})$ dissolved in $\mathrm{CDCl}_{3}(0.7 \mathrm{~mL})$ and cholesteryl acetate $(100 \mathrm{mmol})$ dissolved in $\mathrm{CDCl}_{3} .2 \mathrm{D}{ }^{1} \mathrm{H}-{ }^{13} \mathrm{C}$ LR-HSQC and LRHTQC experiments were recorded using a recycle delay of $1 \mathrm{~s}$. The duration of each gradient was $\delta=1 \mathrm{~ms}$, the gradient recovery was $0.2 \mathrm{~ms}$. The 2D data were acquired using 128 data points in the indirect dimension and each spectrum was recorded using 4096 data points. Prior to Fourier transformation, zero filling to 2048 points in F1, and weighting with a sine-squared function were applied. Experimental parameters: acquisition time: $0.42 \mathrm{~s}$, spectral width of $12 \mathrm{ppm}$; relaxation delay: $1 \mathrm{~s}$; number of scans NS = 8; total experimental time: $25 \mathrm{~min}$. Note that the LR-HSQC and LR-HTQC spectra presented in this work were recorded in magnitude mode. Of course, the pulse sequence can be modified and the experiments recorded in phase-sensitive mode using echo-antiecho detection. 


\section{References}

[1] E. Kupce, R. Freeman, Magn. Reson. Chem. 2010, 48, 333-336.

[2] A. V. Buevich, R. T. Williamson, G. E. Martin, J. Nat. Prod. 2014, 77, 1942-1947.

[3] L. Castanar, J. Sauri, R. T. Williamson, A. Virgili, T. Parella, Angew. Chem. Int. Ed. 2014, 53, 8379-8382.

[4] R. T. Williamson, A. V. Buevich, G. E. Martin, Tetrahedron Lett. 2014, 55, 33653366.

[5] L. Castanar, T. Parella, in Annual Reports on NMR Spectroscopy Vol. 84 (Ed.: G. A. Webb), 2015, pp. 163-232.

[6] M. Elyashberg, Trac-Trends in Analytical Chemistry 2015, 69, 88-97.

[7] J. Sauri, M. Frederich, A. T. Tchinda, T. Parella, R. T. Williamson, G. E. Martin, J. Nat. Prod. 2015, 78, 2236-2241.

[8] J. Sauri, G. E. Martin, J. Furrer, Concepts Magn. Reson. 2015, 44A, 227-251.

[9] J. Sauri, Y. Z. Liu, T. Parella, R. T. Williamson, G. E. Martin, J. Nat. Prod. 2016, 79, 1400-1406.

[10] G. E. Martin, A. S. Zektzer, Magn. Reson. Chem. 1988, 26, 631-652.

[11] G. E. Martin, R. C. Crouch, J. Nat. Prod. 1991, 54, 1-70.

[12] G. E. Martin, C. E. Hadden, J. Nat. Prod. 2000, 63, 543-585.

[13] A. M. Torres, W. A. Bubb, P. W. Kuchel, J. Magn. Reson., Ser A 2002, 156, 249-257.

[14] A. Bax, M. F. Summers, J. Am. Ceram. Soc. 1986, 108, 2093-2094.

[15] G. Bodenhausen, D. Ruben, Chem. Phys. Lett. 1980, 69, 185-189.

[16] R. Marek, L. Kralik, V. Sklenar, Tetrahedron Lett. 1997, 38, 665-668.

[17] R. T. Williamson, B. L. Marquez, W. H. Gerwick, K. E. Kover, Magn. Reson. Chem. 2000, 38, 265-273.

[18] K. A. Blinov, A. V. Buevich, R. T. Williamson, G. E. Martin, Organic \& Biomolecular Chemistry 2014, 12, 9505-9509.

[19] R. T. Williamson, A. V. Buevich, G. E. Martin, T. Parella, J. Org. Chem. 2014, 79, 3887-3894.

[20] J. Sauri, S. T. S. Chan, A. V. Buevich, K. R. Gustafson, R. T. Williamson, G. E. Martin, Planta Med. 2015, 81, 914-914.

[21] J. Sauri, N. Marco, R. T. Williamson, G. E. Martin, T. Parella, Journal of Magnetic Resonance 2015, 258, 25-32. 
[22] P. Bigler, J. Furrer, Magn. Reson. Chem. 2019, 57, 129-143.

[23] K. Motiram-Corral, P. Nolis, J. Sauri, T. Parea, J. Nat. Prod. 2020, 83, 12751282.

[24] P. Bigler, J. Furrer, Magn. Reson. Chem. 2018, 56, 1101-1116.

[25] J. M. Schmidt, H. Rueterjans, Journal of the American Chemical Society 1990, 112, 1279-1280.

[26] J. M. Schmidt, H. Thüring, A. Werner, H. Rüterjans, R. Quaas, U. Hahn, Eur. J. Biochem. 1991, 197, 643-653.

[27] D. M. Doddrell, D. T. Pegg, M. R. Bendall, Journal of Magnetic Resonance 1982, 48, 323-327.

[28] H. Kessler, P. Schmieder, M. Köck, M. Reggelin, Journal of Magnetic Resonance (1969) 1991, 91, 375-379.

[29] M. Liu, R. D. Farrant, J. C. Lindon, J. K. Nicholson, Magn. Reson. Chem. 1995, 33, 212-219.

[30] P. Schmieder, M. Leidert, M. Kelly, H. Oschkinat, Journal of Magnetic Resonance 1998, 131, 199-202.

[31] M. Schubert, M. Smalla, P. Schmieder, H. Oschkinat, Journal of Magnetic Resonance 1999, 141, 34-43.

[32] J. P. Marino, J. L. Diener, P. B. Moore, C. Griesinger, Journal of the American Chemical Society 1997, 119, 7361-7366.

[33] J. M. Bohlen, G. Bodenhausen, Journal of Magnetic Resonance Series A 1993, 102, 293-301.

[34] R. R. Ernst, G. Bodenhausen, A. Wokaun, Principles of nuclear magnetic resonance in one and two dimensions, Clarendon Press, Oxford, 1987.

[35] J. Keeler, Understanding NMR Spectroscopy, Wiley, 2011.

[36] R. Hurd, J. Magn. Reson. 1990, 87, 422-428.

[37] R. E. Hurd, B. K. John, Journal of Magnetic Resonance 1991, 91, 648-653.

[38] T. E. Burrow, R. G. Enriquez, W. F. Reynolds, Magn. Reson. Chem. 2009, 47, 1086-1094. 